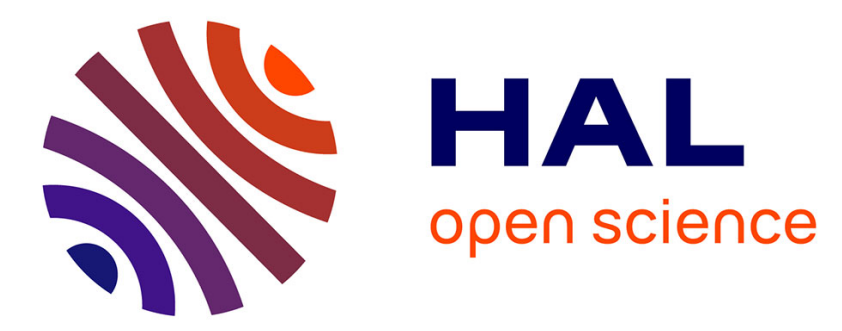

\title{
Surface tension of spherical drops from surface of tension
} Ahmed-Amine Homman, Emeric Bourasseau, Gabriel Stoltz, Patrice Malfreyt, Loic Strafella, Aziz Ghoufi

\section{To cite this version:}

Ahmed-Amine Homman, Emeric Bourasseau, Gabriel Stoltz, Patrice Malfreyt, Loic Strafella, et al.. Surface tension of spherical drops from surface of tension. Journal of Chemical Physics, 2014, 140 (3), pp.034110. 10.1063/1.4862149 . hal-00932843

\section{HAL Id: hal-00932843 https://hal.science/hal-00932843}

Submitted on 17 Apr 2018

HAL is a multi-disciplinary open access archive for the deposit and dissemination of scientific research documents, whether they are published or not. The documents may come from teaching and research institutions in France or abroad, or from public or private research centers.
L'archive ouverte pluridisciplinaire HAL, est destinée au dépôt et à la diffusion de documents scientifiques de niveau recherche, publiés ou non, émanant des établissements d'enseignement et de recherche français ou étrangers, des laboratoires publics ou privés. 


\title{
Surface tension of spherical drops from surface of tension
}

\author{
A.-A. Homman, ${ }^{1}$ E. Bourasseau, ${ }^{1}$ G. Stoltz, ${ }^{2}$ P. Malfreyt,${ }^{3}$ L. Strafella, ${ }^{4}$ and A. Ghoufii ${ }^{4}$, a) \\ ${ }^{1}$ CEA/DAM DIF, F-91297 Arpajon Cedex, France \\ ${ }^{2}$ Université Paris-Est, CERMICS (ENPC), INRIA, F-77455 Marne-la-Vallée, France \\ ${ }^{3}$ Institut de Chimie de Clermont-Ferrand, Université Blaise Pascal, UMR CNRS 6296, ICCF, BP 10448, \\ F-63000 Clermont-Ferrand, France \\ ${ }^{4}$ Institut de Physique de Rennes, Université de Rennes 1 UMR 6251 CNRS, 263 avenue Général Leclerc, 35042 \\ Rennes, France
}

(Received 21 November 2013; accepted 1 January 2014; published online 17 January 2014)

\begin{abstract}
The determination of surface tension of curved interfaces is a topic that raised many controversies during the last century. Explicit liquid-vapor interface modelling (ELVI) was unable up to now to reproduce interfacial behaviors in drops due to ambiguities in the mechanical definition of the surface tension. In this work, we propose a thermodynamic approach based on the location of surface of tension and its use in the Laplace equation to extract the surface tension of spherical interfaces from ELVI modelling. ( 2014 AIP Publishing LLC. [http://dx.doi.org/10.1063/1.4862149]
\end{abstract}

\section{INTRODUCTION}

Molecular simulation has became a powerful method in the last decade to quantitatively predict the surface tension of complex fluids $(\gamma){ }^{1,2}$ Whereas the calculation of $\gamma$ for planar geometries is now under control, many controversies remain about the dependence of the surface tension on the drop size, and the validity of the mechanical definition of the local pressure ${ }^{3-7}$ resulting from the arbitrariness of the choice of contour to define the pairwise interactions. This leads to an ambiguity in the definition of the local elements of the pressure tensor in the spherical geometry. Despite this ambiguity, two mechanical methods are widespreadly used in molecular simulation of explicit liquid-vapor interface (ELVI): the Irving-Kirkwood $(\mathrm{IK})^{8}$ and the Harasima $(\mathrm{H})^{9}$ approaches. On the contrary, the density functional theory (DFT) calculations allow an accurate and rigorous estimation of $\gamma(R)$ with respect to the drop size $(R))^{5,7}$ From the IK method, the following tendencies were found: (i) a monotonic increase of $\gamma$ with respect to the drop size while a non-monotonic behavior was observed from DFT; (ii) the values of $\gamma$ significantly disagree with the DFT results; and (iii) a positive Tolman length $(\delta)$ was found while DFT calculations predict a negative value. These disagreements were systematically observed over the last 30 years. Up to now, no molecular simulation of the explicit liquid-vapor interface was able to recover the DFT trends. ${ }^{10}$ Indeed, Binder et al. ${ }^{11}$ used a thermodynamic analysis of two-phase coexistence in finite boxes at fixed total density to extract the surface tension. Although the DFT method allows a direct and unambiguous calculation of $\gamma$, its application to realistic systems with complex interactions is more difficult. Conversely, molecular simulation is capable to fully take into account the complexity in the interactions, but the calculation of surface tension from molecular simulation of the ELVI remains ambiguous.

In this work, we present a general thermodynamic route to calculate the surface tension of spherical drops and Tol-

\footnotetext{
a)Electronic mail: aziz.ghoufi@univ-rennes1.fr
}

man length from molecular simulations of the ELVI. Our route is based on the location of the surface of tension (which is the radius at which the tension acts ${ }^{3}$ ) and its use in the Laplace equation. Indeed, if the radius of surface of tension is known, Gibbs, ${ }^{12}$ Hill $^{13}$, and Rowlinson and Widom ${ }^{3}$ have clearly established that the calculation of surface tension of spherical interfaces is possible through the Laplace relation $\gamma_{s}=\frac{R_{s}(\Delta P)}{2}$, where $R_{s}$ is the radius of surface of tension, $\gamma_{s}$ the surface tension at $R_{s}$, and $\Delta P$ the pressure difference between the vapor and liquid phases separated by the surface of tension. Up to now, the surface of tension was determined from the Laplace equation once the surface tension was calculated. In this work we follow the thermodynamics development of Hill ${ }^{13}$ and present a new approach to calculate $\gamma_{s}$ based on the extraction of $R_{s}$ from molecular simulations.

\section{THERMODYNAMICS AND STATISTICAL MECHANICS BACKGROUNDS}

As shown in Figure 1 the system is modeled by a sphere of liquid $(\alpha)$ surrounded by a vapor phase $(\beta)$.

The spherical drop is centered in a cubic box of length $L$. The two phases are deemed to be separated by a dividing surface of radius $R$. Furthermore, the density profile is assumed to reach uniform values at some distance prior to $R_{\alpha}$ and $R_{\beta}$. The normal pressures acting on the surface at $R_{\alpha}$ and $R_{\beta}$ are constant through the liquid and vapor phases, respectively. Using this model, and following, for example, Rowlinson and Widom, ${ }^{3}$ the total differential of the Helmholtz free energy can be expressed as

$$
d F=-p_{\alpha} d V_{\alpha}-p_{\beta} d V_{\beta}+\gamma d A+C d R-S d T+\sum_{i} \mu_{i} d N_{i},
$$

where $p_{\alpha}$ and $p_{\beta}$ are the pressures of the $\alpha$ and $\beta$ phases, $A$ is the area located at $R, \gamma$ is the surface tension at $R, C$ is the coefficient of the curvature terms, $\mu_{i}$ is the chemical potential of particle $i, S$ is the entropy, and $T$ is the temperature. From 


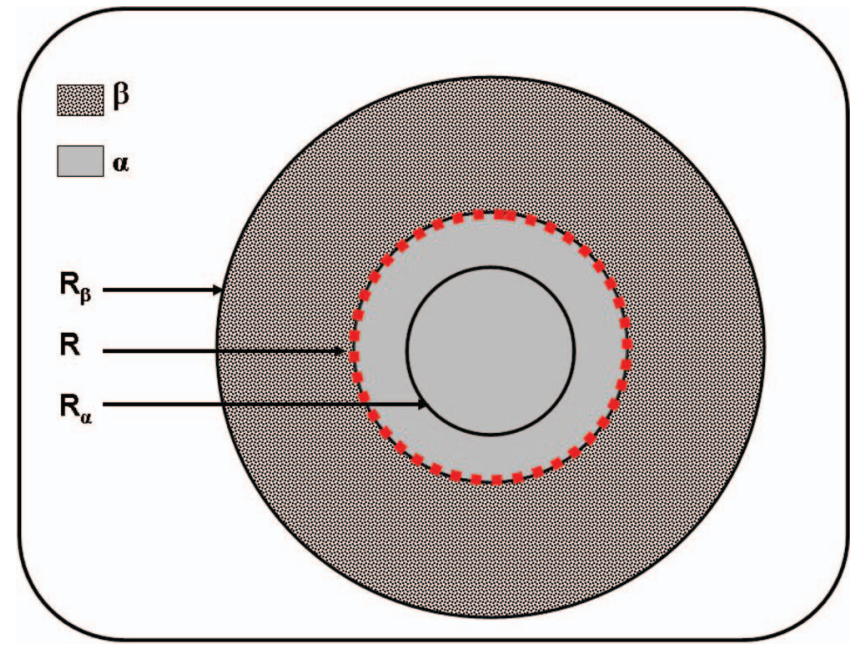

FIG. 1. Schematic picture of the system under consideration, with some key variables.

the model defined in Figure 1, the following relations can be expressed as $V_{\alpha}=\frac{4 \pi\left(R^{3}-R_{\alpha}^{3}\right)}{3}, V_{\beta}=\frac{4 \pi\left(R_{\beta}^{3}-R^{3}\right)}{3}$, and $A=4 \pi R^{2}$. Thus by differentiating $V_{\alpha}, V_{\beta}$, and $A$ with respect to $R_{\alpha}, R_{\beta}$, Eq. (1) can be written as

$$
\begin{aligned}
d F= & +R_{\alpha}^{2} 4 \pi p_{\alpha} d R_{\alpha}-R_{\beta}^{2} 4 \pi p_{\beta} d R_{\beta}-S d T+\sum_{i} \mu_{i} d N_{i} \\
& +\left[C+8 \pi R \gamma+4 \pi p_{\beta} R^{2}-4 \pi p_{\alpha} R^{2}\right] d R .
\end{aligned}
$$

From Eq. (2) we define the following unambiguous partial derivative:

$$
\left[\frac{\partial F}{\partial R}\right]_{R_{\alpha}, R_{\beta}, N, T}=C+4 \pi R\left[2 \gamma+\left(p_{\beta}-p_{\alpha}\right) R\right] .
$$

The thermodynamic equilibrium can be deduced from the minimality criterion such that

$$
\left.\left[\frac{\partial F}{\partial R}\right]_{R_{\alpha}, R_{\beta}, N, T}\right|_{R=R_{s}}=0,
$$

where $R_{s}$ corresponds to the minimum of the free energy. One of the solutions of Eq. (3) and Eq. (4) is

$$
C\left(R_{s}\right)=0 \text { and } \gamma_{s}=\frac{R_{s}\left(p_{\alpha}-p_{\beta}\right)}{2} .
$$

Thus, we recover the well-known dividing surface condition expressed by Hill et al. ${ }^{13}$ based on the so-called surface of tension of Gibbs ${ }^{12}$ and defined as $C\left(R_{S}\right)=0$, where $R_{s}$ is the position of the surface of tension. At $R_{s}$ the surface of tension is noted $\gamma_{s}$ and the Laplace equation $\left(\gamma_{s}=\frac{R_{s}\left(p_{\alpha}-p_{\beta}\right)}{2}\right)$ must be recovered. ${ }^{3}$ This development provides a thermodynamic evidence of Laplace equation and of the dividing surface condition expressed by Hill and Gibbs. Thus, the problem is reduced to the determination of $R_{s}$. We define to this end one possible transformation, compatible with the canonical ensemble, to evaluate the derivative given in Eq. (3). This transformation is indexed by a test radius of tension (denoted by $R_{t}$ ). We then evaluate all the corresponding free energy derivatives and look for the value $R_{s}$ where the derivative vanishes. We approximate the derivative by a finite difference based on the Free Energy Perturbation (FEP) method, ${ }^{14-16}$ where two states are defined: (i) a reference state (indexed by 0 ) where the position of the surface of tension is $R_{t 0}$; and (ii) a perturbed state (indexed by 1 ) where the perturbed radius of tension is noted $R_{t 1}$. For a small parameter $\varepsilon$, we consider the following volume preserving transformation, which leaves the boundaries of the system invariant $\left(R_{\alpha}, R_{\beta}\right)$ : a particle at Cartesian coordinates $\left\{x_{i}, y_{i}, z_{i}\right\}$ at distance $R_{i}=\sqrt{x_{i}^{2}+y_{i}^{2}+z_{i}^{2}}$ from the origin is displaced in the radial direction as

$$
\begin{cases}R_{i 1}=R_{i 0}\left(1+\varepsilon\left(R_{\alpha}-R_{t}\right)\right)=R_{i 0} \Delta \alpha & \text { if } R_{\alpha} \leq R_{i 0}<R_{t} \\ R_{i 1}=R_{i 0}\left(1+\varepsilon\left(R_{\beta}-R_{t}\right)\right)=R_{i 0} \Delta \beta & \text { if } R_{t} \leq R_{i 0} \leq R_{\beta},\end{cases}
$$

with $\left(1+\varepsilon\left(R_{\alpha}-R_{t}\right)\right)=\Delta \alpha$ and $\left(1+\varepsilon\left(R_{\beta}-R_{t}\right)\right)=\Delta \beta$. Note that one subsystem is expanded while the other one is contracted. Indeed, for $R_{\alpha} \leq R_{i 0}<R_{t}, R_{\alpha}-R_{i 0} \leq 0$ while for $R_{t} \leq R_{i 0} \leq R_{\beta}, R_{\beta}-R_{i 0} \geq 0$. The absolute free energy reads

$$
F=-k_{B} T \ln Q_{N V T}, \quad Q_{N V T}=\frac{V^{N}}{\Lambda^{3 N} N !} \int \mathrm{e}^{-\beta U} d \tau^{N},
$$

where $U\left(\mathbf{r}^{N}\right)$ is the configurational energy of the system and $d \tau=d r_{x} d r_{y} d r_{z}$, the infinitesimal volume. $r_{x}, r_{y}$, and $r_{z}$ are the positions of particles. Our transformation divides the system in three parts: (1) the space of phase which is invariant by perturbation $\left(\mathrm{Q}_{I}\right.$ is the associated partition function), (2) the contracted space of phase $\left(\mathrm{Q}_{I I}\right.$ is the associated partition function), and (3) the expanded phase $\left(\mathrm{Q}_{I I I}\right)$. The total partition function in the reference state is then expressed as $Q_{N V T}^{0}=Q_{I}^{0} Q_{I I}^{0} Q_{I I I}^{0}$. The free energy derivative is then approximated as

$$
\begin{aligned}
\left|\frac{\partial F}{\partial R}\right|_{R=R_{t}} \simeq \frac{\Delta F\left(R_{t}\right)}{R_{t} \varepsilon} & =-\frac{k_{B} T}{R_{t} \varepsilon} \ln \frac{Q_{N V T}^{1}}{Q_{N V T}^{0}} \\
& =-\frac{k_{B} T}{R_{t} \varepsilon} \ln \frac{Q_{I}^{1} Q_{I I}^{1} Q_{I I I}^{1}}{Q_{I}^{0} Q_{I I}^{0} Q_{I I I}^{0}}
\end{aligned}
$$

The ratio of partition function can be expressed as function of $d \tau_{I}, d \tau_{I I}$, and $d \tau_{I I I}$

$$
\frac{Q_{I}^{1} Q_{I I}^{1} Q_{I I I}^{1}}{Q_{I}^{0} Q_{I I}^{0} Q_{I I I}^{0}} \propto \frac{\int\left[d \tau_{I}^{1}\right]^{N_{I}}\left[d \tau_{I I}^{1}\right]^{N_{I I}}\left[d \tau_{I I I}^{1}\right]^{N_{I I I}}}{\int\left[d \tau^{0}\right]^{N}},
$$

with $\left[d \tau^{0}\right]^{N}=\left[d \tau_{I}^{0}\right]^{N^{I}}\left[\tau_{I I}^{0}\right]^{N^{I I}}\left[d \tau_{I I I}^{0}\right]^{N^{I I I}} . N_{I}, N_{I I}$, and $N_{I I I}$ are the number of particles of regions (I), (II), and (III), respectively, such that $N=N_{I}+N_{I I}+N_{I I I}$. Given the expression in Eq. (6) the Cartesian components of position can be written as $r_{\eta}^{1}=r_{\eta}^{0} \Delta_{\xi}$ with $\eta=x, y, z$ and $\xi=\alpha, \beta$. Thus, by differentiating we obtain $d r_{\eta}^{1}=d r_{\eta}^{0} \Delta_{\xi}$, i.e., $d \tau_{I}^{1}=d \tau_{I}^{0}$, $d \tau_{I I}^{1}=d \tau_{I I}^{0} \Delta_{\alpha}^{3}, d \tau_{I I I}^{1}=d \tau_{I I I}^{0} \Delta_{\beta}^{3}$ and Eq. (10) can be expressed as

$$
\frac{Q_{I}^{1} Q_{I I}^{1} Q_{I I I}^{1}}{Q_{I}^{0} Q_{I I}^{0} Q_{I I I}^{0}} \propto \frac{\int\left[d \tau^{0}\right]^{N} \Delta_{\alpha}^{3 N_{I I}} \Delta_{\beta}^{3 N_{I I I}}}{\int\left[d \tau^{0}\right]^{N}}
$$


TABLE I. Physical properties of nanodroplets. $N$ is the number of particles, $R_{e}$ the Gibbs radius, $R_{s}$ the radius of surface of tension, and $\Delta p$ the pressure difference between liquid and vapor phases. The number in brackets denotes the uncertainty in the last digit.

\begin{tabular}{rrrcccccc}
\hline \hline \multicolumn{1}{c}{$R_{e}$} & \multicolumn{1}{c}{$R_{s}$} & \multicolumn{1}{c}{$N$} & \multicolumn{1}{c}{$L$} & \multicolumn{1}{c}{$p_{l}$} & \multicolumn{1}{c}{$p_{g}$} & $\Delta p$ & $\delta(R)=R_{e}-R_{s}$ & $\gamma_{s}$ \\
\hline 3.96 & $4.52(2)$ & 484 & 20 & $0.20(1)$ & $0.0210(2)$ & 0.178 & -0.54 & $0.4023(6)$ \\
5.94 & $6.42(2)$ & 960 & 20 & $0.15(2)$ & $0.0183(1)$ & 0.133 & -0.46 & $0.4269(4)$ \\
6.60 & $7.11(2)$ & 1662 & 30 & $0.13(1)$ & $0.0178(1)$ & 0.118 & -0.52 & $0.4195(5)$ \\
10.02 & $10.16(4)$ & 3658 & 30 & $0.10(2)$ & $0.0166(2)$ & 0.081 & -0.08 & $0.4115(9)$ \\
16.22 & $16.32(3)$ & 15660 & 50 & $0.071(8)$ & $0.0156(3)$ & 0.049 & -0.12 & $0.4036(7)$ \\
\hline \hline
\end{tabular}

which involves

$$
\begin{aligned}
\left|\frac{\partial F}{\partial R}\right|_{R=R_{t}} \simeq & \frac{\Delta F\left(R_{t}\right)}{R_{t} \varepsilon}=-\frac{k_{B} T}{R_{t} \varepsilon} \ln \frac{Q_{N V T}^{1}}{Q_{N V T}^{0}} \\
= & -\frac{k_{B} T}{R_{t} \varepsilon} \ln \left\langle\Delta_{\alpha}^{3 N_{I I}} \Delta_{\beta}^{3 N_{I I I}}\right. \\
& \left.\times \exp \left(-\left[\frac{U\left(\mathbf{r}^{1}(N)\right)-U\left(\mathbf{r}^{0}(N)\right)}{k_{B} T}\right]\right)\right\rangle .
\end{aligned}
$$

In practice, we average over the estimated derivatives obtained with $\pm \varepsilon$ in order to reduce statistical errors. To summarize, $R_{s}$ corresponds to the value of $R_{t}$ such that $\Delta F\left(R_{s}\right)=0$. The extraction of the surface tension from Laplace's equation follows once $\Delta p=p_{l}-p_{v}$ is computed. We use the nonexponential thermodynamic perturbation method to compute the local pressure. ${ }^{17}$ As shown in Ref. 18, the ambiguity on the location of pairwise interactions only influences the interfacial region while the bulk liquid and vapor phases are not impacted.

Very interestingly, our approach can also be used to extract the Tolman length $(\delta)$, which is a measure of the deviation of the surface tension with respect to the planar limit. It can be expressed as the limit in the planar case $(R \rightarrow+\infty)$ of the difference between the surface of tension $R_{s}$ and the Gibbs equimolar dividing surface $R_{e}$ :

$$
\delta=\lim _{R \rightarrow \infty} \delta(R) \approx z_{e}-z_{s}, \quad \delta(R)=R_{e}-R_{s},
$$

where $z_{e}$ and $z_{s}$, respectively, are the positions of the Gibbs equimolar surface and surface of tension of planar interface (upon considering that the normal at the interface is along the $z$ axis). While $z_{e}$ can be obtained by a fit using an error function, ${ }^{1} z_{s}$ is more difficult to extract. A straightforward modification of our previous theoretical framework for planar interfaces allows us to obtain the Tolman length from

$$
\left.\left.\lim _{R \rightarrow \infty}\left(\frac{\partial F}{\partial R}\right)_{V}\right|_{R=R_{s}} \approx\left(\frac{\partial F}{\partial z}\right)_{V}\right|_{z=z_{s}}=0 .
$$

The value $z$ is a test value for the planar interface, and we consider the following transformation, in analogy with Eq. (6):

$$
\begin{cases}z_{i 1}=z_{i 0}\left(1+\varepsilon\left(z_{\alpha}-z_{t}\right)\right) & \text { if } z_{\alpha} \leq z_{i 0}<z_{t}, \\ z_{i 1}=z_{i 0}\left(1+\varepsilon\left(z_{\beta}-z_{t}\right)\right) & \text { if } z_{t} \leq z_{i 0} \leq z_{\beta} .\end{cases}
$$

\section{COMPUTATIONAL PROCEDURE}

\section{A. Lennard-Jones model}

In order to consistently compare our results to published works, ${ }^{7}$ we perform Monte Carlo (MC) simulations of the liquid-vapor spherical interface of Lennard-Jones (LJ) particles from a shifted truncated spherically potential (STS) at $T=0.8$ and $r_{c}=2.5$ :

$$
\begin{gathered}
U_{\mathrm{STS}}(r)=4\left(\left(\frac{1}{r}\right)^{12}-\left(\frac{1}{r}\right)^{6}\right), \\
U_{\mathrm{STS}}(r)=U_{\mathrm{STS}}(r)-U_{\mathrm{STS}}\left(r_{c}\right) \text { if } r \leq r_{c}, \\
U_{\mathrm{STS}}(r)=0 \text { if } r>r_{c},
\end{gathered}
$$

where $r$ is the reduced distance, and $T$ and $r_{c}$ are the reduced temperature and the cutoff radius, respectively. The simulation cell is cubic. Box lengths $(L)$ are given in Table I. The liquid-vapor nanodrops are carved from an equilibrated liquid-bulk configuration. Number of particles is given in Table I. Periodic boundary conditions are applied in the three directions. All the results of this paper are given in reduced units. MC simulations of liquid-vapor are carried out in NVT ensemble using $5 \times 10^{9} \mathrm{MC}$ steps of equilibration and $5 \times 10^{9}$ MC steps of acquisition. The maximum displacement is adjusted during the equilibration phase to give an acceptance ratio of 0.4. Calculations were carried out with $\epsilon=1 \times 10^{-5}$.

\section{B. Water liquid-vapor and water-octane spherical interfaces}

To compare with the results obtained by Zakharov et al. ${ }^{19}$ from the mechanical route the TIP4P water model was considered. ${ }^{20}$ The Transferable Potentials for Phase Equilibria (TraPPE) force field ${ }^{21}$ was used to model octane. The description of the interactions can be found in Refs. 2 and 21. Molecular dynamics simulation of the liquid-vapor water nanodroplets was performed in the canonical statistical ensemble using the Hoover's thermostat ${ }^{22}$ with periodic boundary conditions at $298 \mathrm{~K}$. Spherical water-octane interface was generated by surrounding a spherical water droplet with octane. Simulation of water-octane phase was carried out in the NpT ensemble from the Nose-Hoover barostat. ${ }^{23}$ We set up our simulation box in the $x, y, z$ directions of 50,50 , and $50 \AA$, 


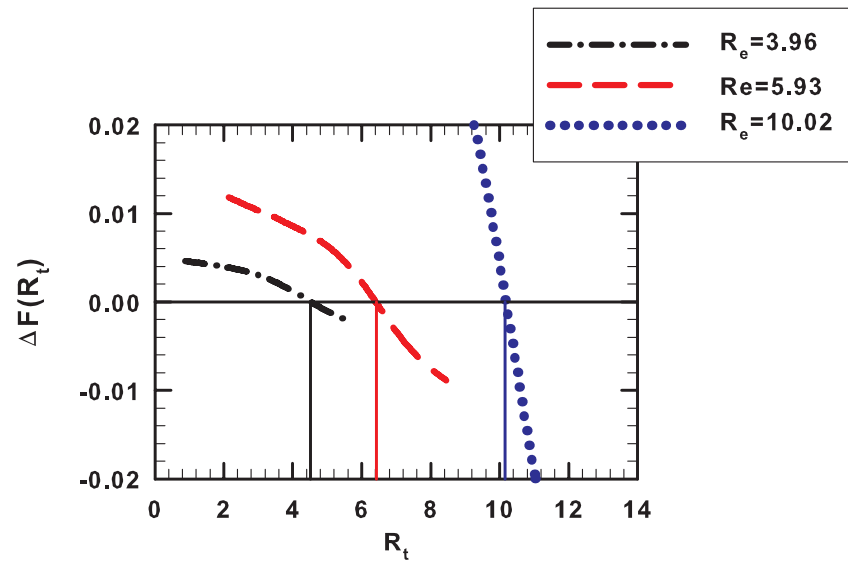

FIG. 2. Free energy difference $(\Delta F)$ as function of the trials radii $R_{t}$ for various drops of Gibbs equimolar radius $R_{e}$. The radius of surface of tension $R_{s}$ is the value such that $\Delta F\left(R_{s}\right)=0$. The droplets are made of LJ particles. Results are given in reduced units.

respectively. A cutoff of $12 \AA$ was used to model the LennardJones interactions. The electrostatic interactions were computed from the Ewald summation..$^{23}$ The equations of motion were integrated using the velocity Verlet algorithm ${ }^{23}$ with a time step of $2 \mathrm{fs}$. Data analysis was performed for the last $10 \mathrm{~ns}$ of simulations after $5 \mathrm{~ns}$ of equilibration. All simulations were carried out from a modified DL-POLY package. ${ }^{24}$

\section{RESULTS AND DISCUSSION}

We report in Figure 2 the free energy difference as function of $R_{t}$. Independently of the drop size, an intersection between $\Delta F\left(R_{t}\right)$ and 0 is observed. This clearly establishes a numerical evidence of our theoretical framework.

In Figure 3 we report the surface tension of LennardJones particles with respect to the drop radius by using different definitions. We observe that the fundamental measure theory (FMT) DFT method provides a surface tension higher than the planar limit $\left(\gamma_{\infty}\right)$ from $R_{e}=4$ and a non-monotonic curvature dependence in $\gamma$. From the mechanical definition of the surface tension (IK definition), the curvature dependence in surface tension is monotonic. Additionally, this approach

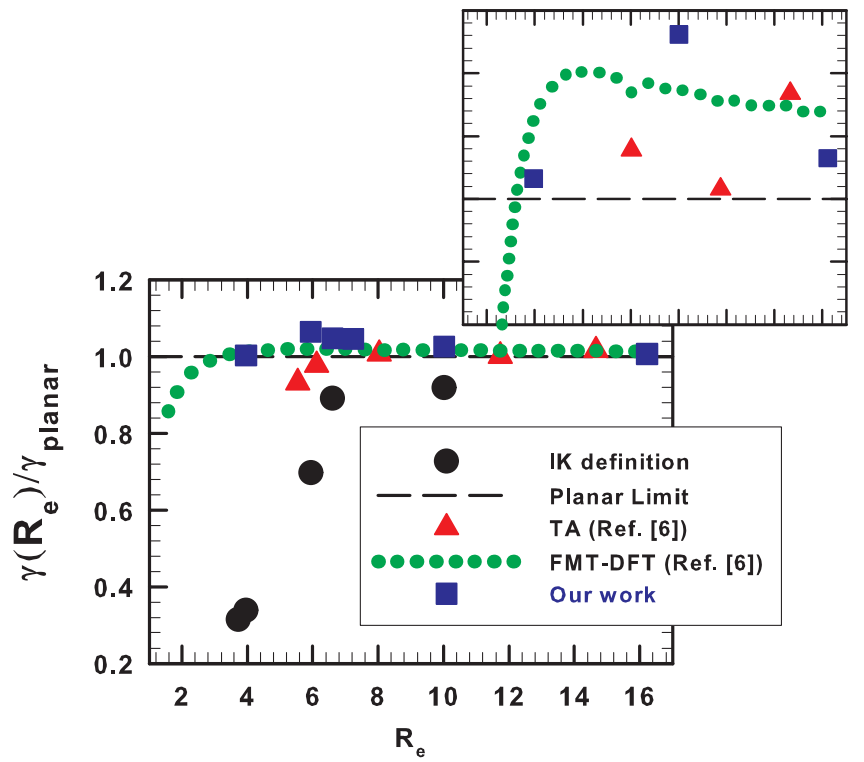

FIG. 3. Normalised surface tension of Lennard-Jones particles with truncated shifted potential $\left(T=0.8\right.$, cutoff $\left.\mathrm{r}_{c}=2.5\right)$ with respect to the drop radius and different definitions. $\gamma_{\text {planar }}$ corresponds to the planar surface tension. TA corresponds to the test area method ${ }^{6}$ and FMT-DFT is a non-local density functional theory. In upper part an enlargement is given to highlight the non-monotonic behavior. Results are given in reduced units.

predicts $\gamma<\gamma_{\infty}$. The method of Sampayo et al. ${ }^{6}$ based on elliptic test-area transformation provides a non-monotonic behavior and $\gamma\left(R_{e}\right)<\gamma_{\infty}$ while the absolute values in surface tension are slightly underestimated with respect to the FMT-DFT values. From our approach a similar tendency to FMT-DFT is found. Indeed, $\gamma$ is larger than $\gamma_{\infty}$ and a nonmonotonic curvature dependence in $\gamma$ is observed. This result shows that the surface tension can be computed from a direct molecular simulation. Interestingly our results are in line with those of Block et al.,${ }^{25}$ van Giessen and Blokhuis, ${ }^{26}$ and Blokhuis and van Giessen ${ }^{27}$ which obtained surface tension from a thermodynamic analysis of two phases coexistence in finite boxes at fixed total density.

The error bar of surface tension calculation was computed by differentiating Eq. (5) and is based on uncertainty of liquid and vapor pressures and the location of surface of tension. As shown in Table I uncertainties on pressure, surface tension, and location of surface of tension are small. Error
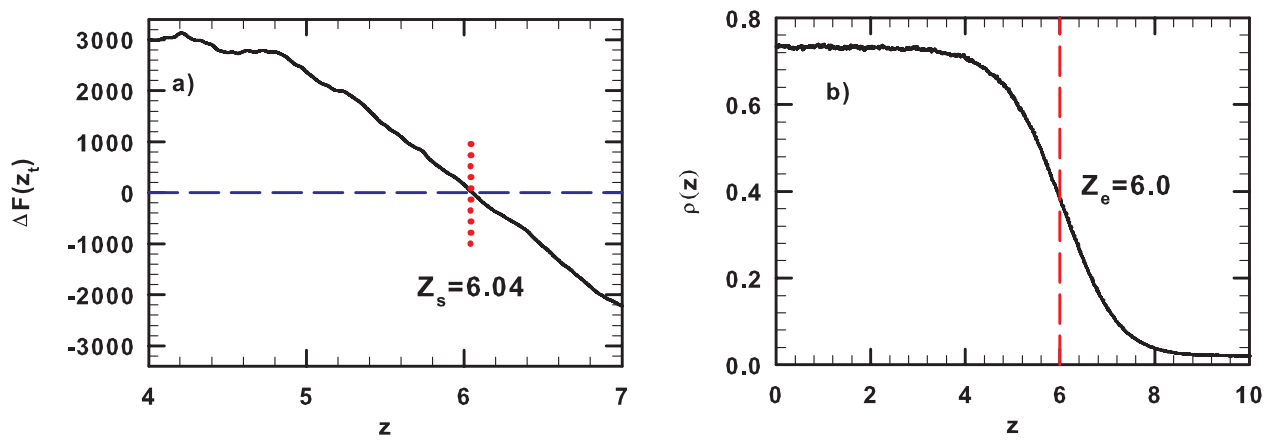

FIG. 4. (a) Free energy difference as function of $z$. The value $z_{s}$ corresponds to $\Delta F\left(z_{s}\right)=0$. (b) Density profile as a function of $z$ for a liquid-vapor system of Lennard-Jones particle at $\mathrm{T}=0.8$ with a cutoff radius of 2.5 . Results are given in reduced units. 
TABLE II. Physical properties of water nanodroplets. $N$ is the number of particles, $R_{e}$ the Gibbs radius, $R_{s}$ the radius of surface of tension, $\delta(R)$ $=R_{e}-R_{s}$ is the Tolman length, and $\Delta p$ the pressure difference between the liquid and the vapor phase. Surface tension of TIP4P water liquid-vapor planar interface is $56.2 \mathrm{mN} \mathrm{m}^{-1}$ without including the long range corrections. Pressure in liquid and vapor phases was computed from the thermodynamic route $^{17}$ and validated from a comparison with the mechanical definition. ${ }^{18}$

\begin{tabular}{rrrrrr}
\hline \hline $\begin{array}{c}R_{e} \\
(\AA)\end{array}$ & $\begin{array}{c}R_{s} \\
(\AA)\end{array}$ & $N$ & $\begin{array}{c}\Delta p \\
(\mathrm{Mpa})\end{array}$ & $\begin{array}{c}\delta(R) \\
(\AA)\end{array}$ & $\begin{array}{c}\gamma \\
\left(\mathrm{mN} \mathrm{m}^{-1}\right)\end{array}$ \\
\hline \multicolumn{7}{c}{ Water liquid-vapor } \\
7.2 & 7.3 & 71 & 188.1 & -0.1 & 68.6 \\
8.4 & 10.3 & 137 & 114.3 & -1.9 & 58.9 \\
11.6 & 12.0 & 235 & 84.3 & -0.4 & 50.6 \\
& \multicolumn{7}{c}{ Water-octane } \\
9.6 & 9.8 & $137-409$ & 116.2 & -0.2 & 55.7 \\
\hline \hline
\end{tabular}

bars on pressures of liquid and vapor phases were computed from the radial profile and are reported in Table I. This accuracy is due to a longer simulation time $\left(5 \times 10^{9} \mathrm{MC}\right.$ steps $)$ which allowed us to get a stable radial pressure in bulk phases and an accurate location of surface of tension.

To confirm the correctness of our approach, we compute the Tolman length of a planar interface based on Eq. (14). Details of computational procedure of planar simulations can be found in Ref. 28. We report in Figure 4(a) the free energy difference $(\Delta F)$ as function of the trials position of surface of tension. The intersection between $\Delta F\left(z_{t}\right)$ and 0 occurs at $z_{s}=6.04$. In Figure 4(b) we provide the density profile and the position of the Gibbs equimolar surface obtained from a fit with an error function $\left(z_{e}=6.0\right)$. Therefore, the Tolman length is $\delta=-0.04 \pm 0.01$. This value is in fair agreement with the DFT calculation obtained by van Giessen and Blokhuis $^{26}$ ( $\left.\delta=-0.1\right)$. Let us note that even with the uncertainty the Tolman length is always negative. The important result is not the absolute value of $\delta$ but rather its sign. Indeed, only Sampayo et $a .^{6}$ have been capable to reproduce a negative Tolman length from molecular simulation of ELVI.

Eventually, we test our calculation on water nanodrops including the dispersion-repulsion and electrostatic interactions. We report in Table II the surface tension and the radius of surface of tension of the water liquid-vapor and wateroctane droplets. The values of $R_{s}$ and $R_{e}$ are very similar, as expected in realistic calculations. Furthermore, we observe a decrease in the surface tension of water drops immersed in liquid octane. This result is consistent with those obtained for planar interfaces. All of these results confirm the soundness of our approach. Thus this method can be easily transferred to multicomponent systems.

\section{CONCLUDING REMARKS}

To summarize, we developed in this work a new thermodynamic path to compute the surface tension of spherical interfaces from the determination of the position of the surface of tension. Indeed, (i) a non monotonic behavior of surface tension is recovered; (ii) the surface tension of nanodrops is in good agreement with the DFT calculations; and (iii) the Tolman length is in line with theoretical route, in sign, and in absolute value.

\section{ACKNOWLEDGMENTS}

The authors are grateful to D. J. Tildesley and G. Jackson for the fruitful discussions.

${ }^{1}$ S. Senapati and M. L. Berkowitz, Phys. Rev. Lett. 87, 176101 (2001).

${ }^{2}$ A. Ghoufi, F. Goujon, V. Lachet, and P. Malfreyt, J. Chem. Phys. 128, 154716 (2008)

${ }^{3}$ J. S. Rowlinson and B. Widom, Molecular Theory of Capillarity (Clarendon Press, Oxford, 1982).

${ }^{4}$ P. Schofield and J. R. Henderson, Proc. R. Soc. London, Ser. A 379, 231 (1982).

${ }^{5}$ E. M. Blokhuis and D. Bedeaux, J. Chem. Phys. 97, 3576 (1992).

${ }^{6}$ J. G. Sampayo, A. Malijevský, E. A. Müller, E. de Miguel, and G. Jackson, J. Chem. Phys. 132, 141101 (2010).

${ }^{7}$ A. Malijevsky and G. Jackson, J. Phys.: Condens. Matter 24, 464121 (2012).

${ }^{8}$ J. G. Kirkwood and F. P. Buff, J. Chem. Phys. 17, 338 (1949).

${ }^{9}$ A. Harasima, Adv. Chem. Phys. 1, 203 (1958).

${ }^{10}$ Y.-A. Lei, T. Bykov, S. Yoo, and X. Zeng, J. Am. Chem. Soc. 127, 15346 (2005).

${ }^{11}$ A. Troster, M. Oettel, B. Block, P. Virnau, and K. Binder, J. Chem. Phys. 136, 064709 (2012).

${ }^{12}$ J. Gibbs, The Collected Works of J. Willard Gibbs (Yale University Press, New Haven, 1948).

${ }^{13}$ T. Hill, J. Chem. Phys. 56, 526 (1952).

${ }^{14}$ W. Zwanzig, J. Chem. Phys. 22, 783 (1954).

${ }^{15}$ A. Ghoufi and P. Malfreyt, Mol. Phys. 104, 2929 (2006)

${ }^{16}$ A. Ghoufi, C. Bonal, J. P. Morel, N. Morel-Desrosiers, and P. Malfreyt, J. Phys. Chem. B 108, 11744 (2004).

${ }^{17}$ A. Ghoufi and P. Malfreyt, J. Chem. Phys. 136, 024104 (2012).

${ }^{18}$ S. M. Thompson and K. E. Gubbins, J. Chem. Phys. 81, 530 (1984).

${ }^{19}$ V. Zakharov, E. Brodskaya, and A. Laaksonen, J. Chem. Phys. 107, 10675 (1997).

${ }^{20}$ W. Jorgensen and J. Madura, Mol. Phys. 56, 1381 (1985).

${ }^{21}$ M. Martin and J. Siepmann, J. Phys. Chem. B 102, 2569 (1998).

${ }^{22}$ W. Hoover, Phys. Rev. A 31, 1695 (1985).

${ }^{23}$ M. P. Allen and D. J. Tildesley, Computer Simulation of Liquids (Oxford University Press, 1987).

${ }^{24}$ T. R. Forester and W. Smith, DLPOLY, CCP5 Program Library, Daresbury Lab., U.K., 1004.

${ }^{25}$ B. Block, S. Das, M. Oettel, P. Virnau, and K. Binder, J. Chem. Phys. 133, 154702 (2010).

${ }^{26}$ A. E. van Giessen and E. M. Blokhuis, J. Chem. Phys. 131, 164705 (2009).

${ }^{27}$ E. M. Blokhuis and A. E. van Giessen, J. Phys.: Condens. Matter 25, 225003 (2013).

${ }^{28}$ A. Ghoufi and P. Malfreyt, Mol. Simul. 39, 603 (2013). 